\title{
SENSITIVITY INVESTIGATION OF THREE-CYLINDER MODEL OF HUMAN KNEE JOINT
}

\author{
István Bíró ${ }^{1}$, Béla M. Csizmadia ${ }^{2}$, Gábor Katona ${ }^{2}$ \\ ${ }^{1}$ University of Szeged, Faculty of Engineering, Szeged, Hungary \\ ${ }^{2}$ Szent István University, Faculty of Mechanical Engineering, Gödöllő, Hungary \\ biro-i@mk.u-szeged.hu
}

\begin{abstract}
The operation is unavoidable in a certain part of patients suffering from arthrosis. The contact surfaces of wide-spread applied human knee joint prostheses can be described with simple geometrical elements. The relative motion realized by knee joint and as its results the stability of the whole body is harmonic ensured by complicated condyle surfaces. For this reason the implanted prostheses comply with requirements limited and it causes additional load on the diseased bony tissue. According to observations at the fastening of the prostheses the bony tissues become inflamed which after some years need new operation.
\end{abstract}

At present it is a general aim in biomechanics to create a better mechanical model of human knee joint which can approach the natural motion and on its basis to make new prostheses. The motion of the human knee joint have been studying by many biomechanical research groups for decades. The problem is very complex and specific from technical point of view. The cause of complexity is partly the elaborateness of elements, partly the typical rheological properties of the components (bones, cartilages and other soft tissues).

Authors as members of the Szent István University Biomechanical Research Group in order to describe the motion of knee joint at first joined coordinate-systems on the basis of anatomical landmarks to the femur and tibia moreover joined a three-cylindrical mechanism as mechanical model to the axes of coordinate-systems.

The aim of the investigation is to determine the six independent kinematical parameters of tibia compared to the fixed femur during flexion and extension. The experimental examinations were carried out on cadaver knees in cooperation with doctors of Szent János Hospital. The positioning was tracked by optical positioning appliance. Needed parameters can be obtained from the recorded data determined by the kinematical model.

Considering the irregular shapes of femur and tibia the anatomical coordinate systems can be joined with more or less position mistake. The aim of this paper is the determination of the effects of position mistakes on kinematical parameters.

Keywords: knee joint; kinematical model; optical positioning; accuracy; sensitivity investigation 


\section{Introduction}

Different constraints enable relative motion of joined rigid bodies referring to each other. The constraints depending on their shapes have one or more degree of freedom. In case of joints the degree of freedom cannot be always determined. It is expedient to treat the human knee joint as six degree of freedom because of its complicated shape. In this case to the precise description of motion achieved by knee joint we need six independent position parameters ${ }^{1,7}$.

In recent years the number of kinematical models of anatomical joints has increased. In case of certain models so many researchers have measured and described joint motion with less than six degrees of freedom. Obviously the treatment of six degree of freedom models is the most difficult. The motion of human knee joint can be described by following components: The flexion-extension is defined around the medio-lateral axis, internal-external rotation around the tibial axis and the abduction-adduction around the anteriorposterior (floating) axis. The medio-lateral translation is measured along the medio-lateral axis, proximal-distal translation along the tibial axis and antero-posterior translation along the mutually perpendicular floating axis.

Description of motion components in such a way a little bit subjective. In order to descript the motion components precisely it is needed to join coordinate-systems to the femur and tibia consequently.

In recent decades to measure kinematical parameters of human knee joint different methods have been developed. In these methods it is measured and processed the motion of markers fastened to femur and tibia referring to each other. In vitro mechanical investigations are mainly phantom or simulated computer models or cadaver motion experiments ${ }^{7}$.
The visual examinations were based on marker technique to sign single points or axis of the extremities delineating their motion. In spite of that the newly introduced techniques developed in an enormous numbers in the last decades e.g. the radiology, fluoroscopy, threedimensional CT, MRI, stereophotogrammetry, ultrasound, etc. most of the results were unreliable, inconsistent with other published data $3,4,5,6$. The range of the tibia out and inrotation along the flexion-extension motion of the knee had been established by different authors as between 5 up to 17 degrees, moreover the character of this diagram is variable $e^{8,9}$. On the basis of difference of published results it is quite difficult to establish exact character concerning the motion of knee joint.

\section{Method}

Authors developed a special appliance ${ }^{10,11,12}$ in order to make a serial experiments. The aim of them was the determination of change of six independent kinematical parameters of tibia (shin bone) compared to femur (thigh bone) during of motion of human knee joint. From recorded data needed parameters can be determined by the aid of kinematical model.

To the presented sensitivity investigation it is necessary to determine anatomical landmarks on femur and tibia moreover coordinate-systems joining to the determined anatomical landmarks. Details of this process can be found in paper of Katona et al $1^{13,14,15}$. Considering the biological characters of femur and tibia the optical positioning of anatomical landmarks can be achieved with more or less position mistakes. The aim of this paper is the determination of the effects on kinematical parameters of position mistakes during flexion-extension of human knee joint.

Authors on the basis of current international standards and conventions (e.g. from the Inter- 


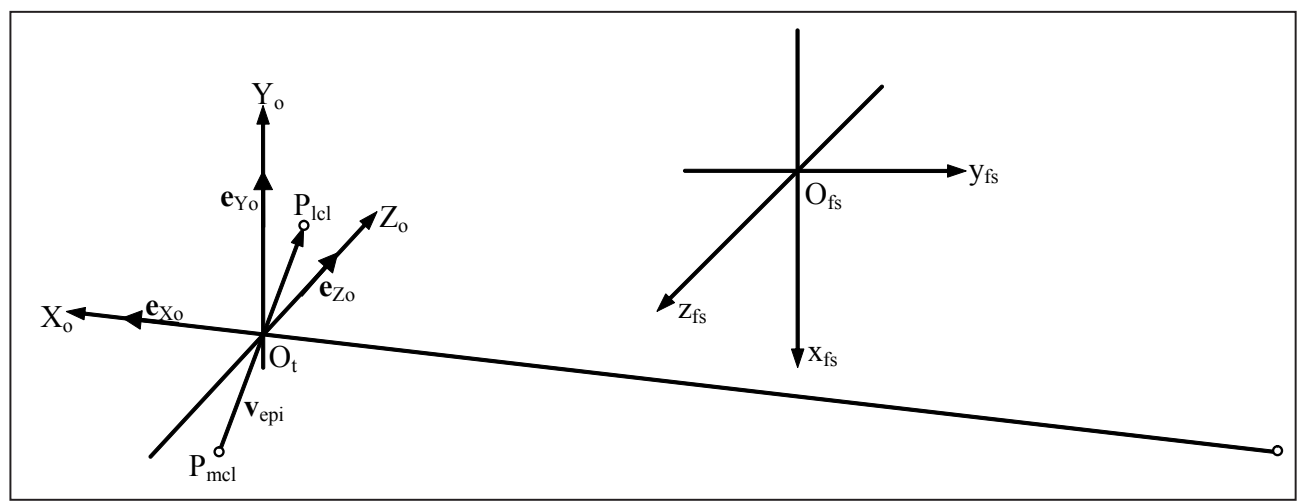

Figure 1. Position of anatomical coordinate-system $X_{o} Y_{o} Z_{o}$ joined to femur in coordinate-system $f_{s}$ (cadaver lying on his back, investigated right leg, view from medial side)

national Society of Biomechanics) ${ }^{16}$ using the above mentioned anatomical landmarks joined coordinate-systems to femur and tibia.

At first to the processing of recorded positioning coordinates it is necessary to take the anatomical coordinate-system on anatomical landmarks of femur in coordinate-system $f_{s}$ ( $f s$ in the appliance, joined to femur rigidly) (Figure 1).

Needed data determining anatomical coordinate-system $X_{o} Y_{o} Z_{o}$ (in coordinate-system $f s$ ): - centre of the femoral head $(f h)$

- medial and lateral epicondyles $\left(P_{m c l}, P_{l c l}\right)$.

The origin of this anatomical coordinate-system $\left(O_{t}\right)$ coincides with middle point of line between medial and lateral epicondyles. Axis $X_{o}$ of coordinate-system is on the line pointed out by points $O_{t}$ and $f h$ (Figure 1 ). Vector $\mathbf{v}_{\text {epi }}$ is between medial and lateral epicondyles.

Unit vectors of axes of coordinate-systems can be obtained as results of following vector operations:

$\mathbf{e}_{X o}=\frac{\mathbf{v}_{O t-f h}}{\left|\mathbf{v}_{O t-f h}\right|}, \quad \mathbf{v}_{e p i}=\mathbf{r}_{P l c l}-\mathbf{r}_{P m c l}, \quad \mathbf{e}_{e p i}=\frac{\mathbf{v}_{e p i}}{\left|\mathbf{v}_{e p i}\right|}$,
$\mathbf{e}_{Y o}=\mathbf{e}_{e p i} \times \mathbf{e}_{X o}, \mathbf{e}_{Z o}=\mathbf{e}_{X o} \times \mathbf{e}_{Y o}$.

Determining coordinate-system joined to anatomical landmarks on tibia: Needed data for coordinate-system $x_{s} y_{s} z_{s}$ (in appliance):

- apex of the head of the fibula ( $h f)$,

- prominence of the tibial tuberosity $(t t)$,

- distal apex of the lateral and medial malleolus $(k b, b b)$.

The origin of the anatomical coordinate-system $\left(O_{s}\right)$ coincides with middle point of line between distal apex of the lateral and medial malleolus. Unit vectors of axes of coordinatesystems can be obtained as results of next vector operations (Figure 2):

$$
\begin{aligned}
& \mathbf{e}_{k b-b b}=\frac{\mathbf{v}_{k b-b b}}{\left|\mathbf{v}_{k b-b b}\right|}, \quad \mathbf{e}_{k b-h f}=\frac{\mathbf{v}_{k b-h f}}{\left|\mathbf{v}_{k b-h f}\right|}, \quad \mathbf{e}_{O s-t t}=\frac{\mathbf{v}_{O s-t t}}{\left|\mathbf{v}_{O s-t t}\right|}, \\
& \mathbf{e}_{x s}=\mathbf{e}_{k b-b b} \times \mathbf{e}_{k b-h f}, \quad \mathbf{e}_{z s}=\mathbf{e}_{x s} \times \mathbf{e}_{O s-t t}, \quad \mathbf{e}_{y s}=\mathbf{e}_{z s} \times \mathbf{e}_{x s} .
\end{aligned}
$$

During motion investigation of knee joint we follow the change of position of coordinatesystem ts joined to tibia compared to coordinate-system fs joined to femur. We had to describe anatomical coordinate-systems in coordinate-systems joined to femur and tibia for the sake of following: in this way the posi- 


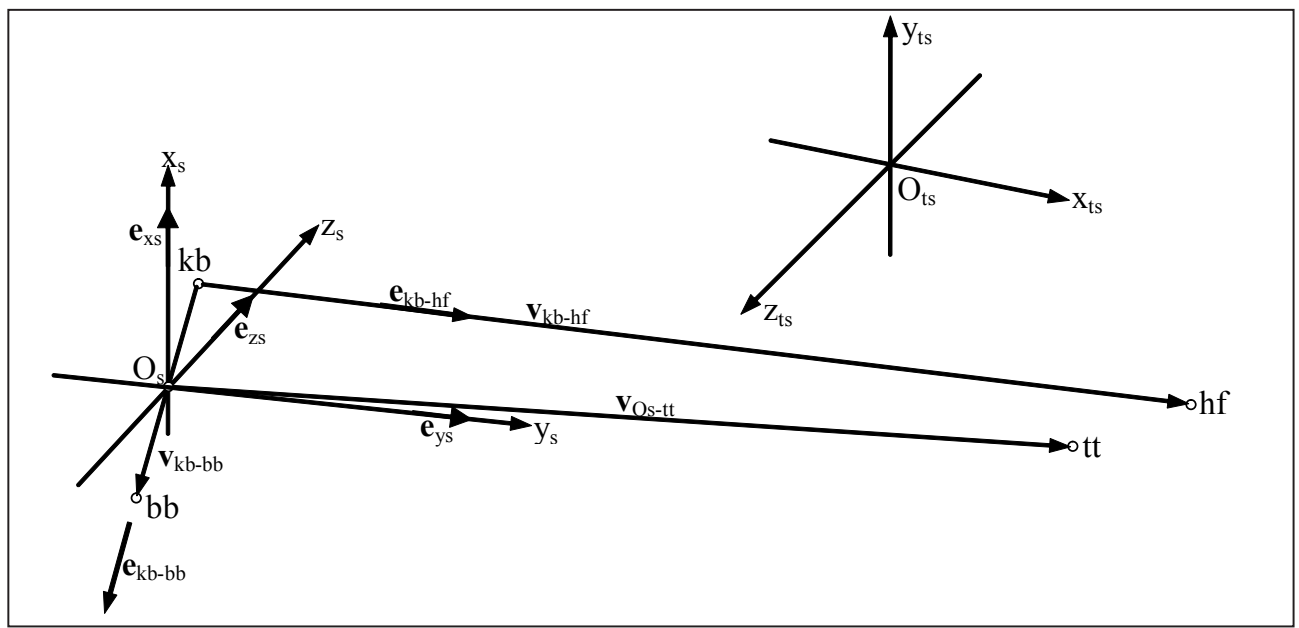

Figure 2. Position of anatomical coordinate-system $x_{s} y_{s} z_{s}$ joined to tibia in coordinate-system $t s$ (cadaver lying on his back, investigated right leg, view from medial side)

tion of anatomical coordinate-system joined to tibia in anatomical coordinate-system joined to femur will be known.

If the anatomical landmarks on femur and tibia are determined with some position mistake the kinematical parameters of knee joint will be modified. These kinematical parameters can be obtained by the aid of three-cylindrical mechanism put in between the above defined anatomical coordinate-systems.

\section{Three-Cylindrical mechanism}

Putting the origin of coordinate-system $X_{3} Y_{3} Z_{3}$ (without modification of direction of axes) into the origin of coordinate-system $x_{s} y_{s} z_{s}$ unit vectors will be the followings:

$\mathbf{e}_{X 3}=\mathbf{e}_{z s}, \quad \mathbf{e}_{Y 3}=-\mathbf{e}_{x s}, \quad \mathbf{e}_{Z 3}=-\mathbf{e}_{y s}$.

The position of coordinate-system $X_{3} Y_{3} Z_{3}$ in the coordinate-system $X_{o} Y_{o} Z_{o}$ is described by the next matrix-equation:

$\left[T_{0-3}\right]=\left[T_{t s-3}\right]\left[T_{a b s-t s}\right]\left[T_{a b s-f s}\right]^{-1}\left[T_{f s-0}\right]^{-1}$ in which the transformation matrices

- $\left[\mathbf{T}_{\mathbf{t s}-\mathbf{3}}\right]$ which contains six position parameters describing the position of anatomical coordinate-system $\left(X_{3} Y_{3} Z_{3}\right)$ joined to tibia in coordinate-system $t s$,

- $\left[\mathbf{T}_{\text {abs-ts }}\right]$ which contains six position parameters describing the position of coordinatesystem $t s$ in absolute coordinate-system (XYZ),

$-\left[\mathbf{T}_{\mathbf{a b s}-\mathbf{f s}}\right]^{-\mathbf{1}}$ inverse transformation matrix which contains six position parameters describing the position of coordinate-system $f_{s}$ in absolute coordinate-system (XYZ),

$-\left[\mathbf{T}_{\mathbf{f s}-\mathbf{0}}\right]^{\mathbf{- 1}}$ inverse transformation matrix which contains six position parameters describing the position of anatomical coordinate-system $X_{o} Y_{o} Z_{o}$ joined to femur in coordinatesystem $f s$.

Transformation matrix $\left[\mathbf{T}_{\mathbf{0}-\mathbf{3}}\right]$ can be written down in another way:

$\left[\mathbf{T}_{0-3}\right]=\left[\begin{array}{ll}\mathbf{e}_{\mathrm{X} 3} & 0 \\ \mathbf{e}_{\mathrm{Y} 3} & 0 \\ \mathbf{e}_{\mathrm{Z} 3} & 0 \\ \mathbf{r}_{\mathrm{O} 3} & 1\end{array}\right]$, 


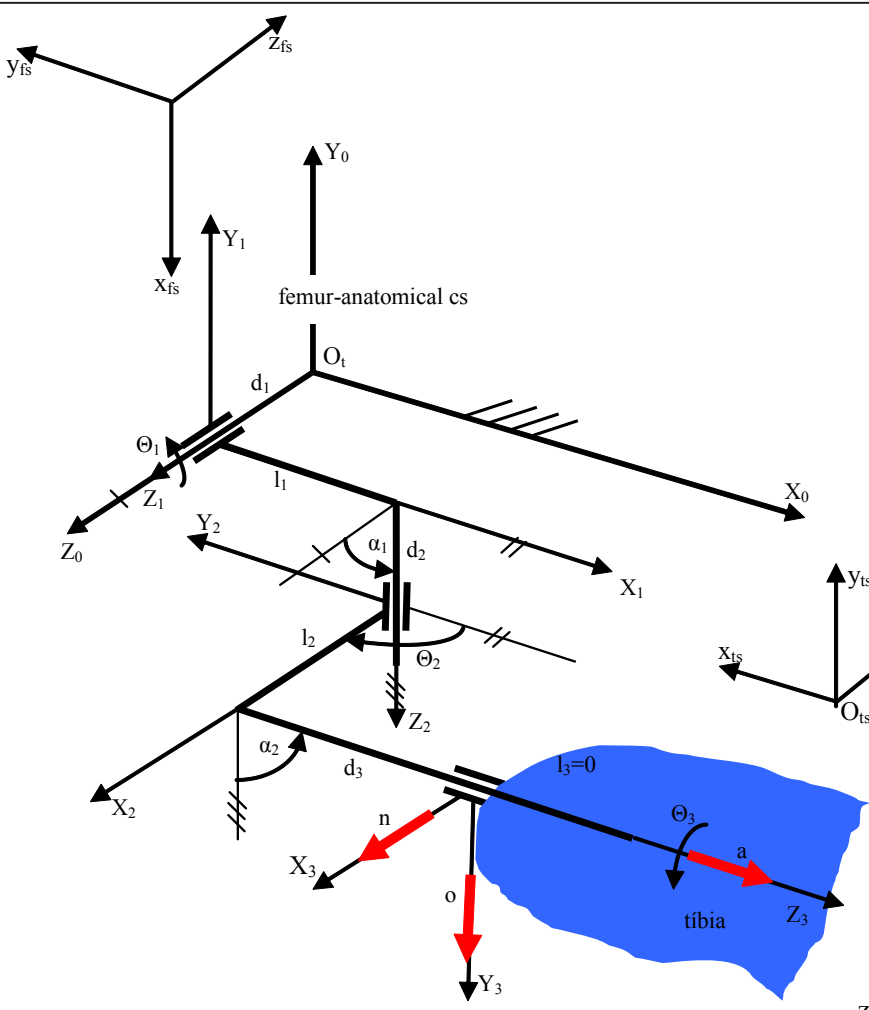

Figure 3. Three-cylindrical mechanism with the defined coordinate-systems (extended position)

where $\mathrm{e}_{X 3}, \mathrm{e}_{\mathbf{Y} 3}, \mathrm{e}_{Z 3}$ are unit vectors of axes $X_{3}$, $Y_{3}, Z_{3}$ in coordinate-system $X_{0} Y_{o} Z_{o}$ and vector $\mathbf{r}_{\mathrm{O} 3}$ contains the coordinates of origin of coordinate-system $X_{3} Y_{3} Z_{3}$ in coordinate-system $X_{o} Y_{0} Z_{0}$.

In case of similar spatial structures the socalled Denavit-Hartenberg $(H D)$ coordinates can be applied (Figure 4). The advantage of the application of HD coordinates: the transformation matrix contains - instead of six four $\left(\Theta_{i}, d_{i}, l_{i}, \alpha_{i}\right)$ variable physical quantities joining to geometrical characters of bodies and their constraint.

In Figure 3 the $H D$ coordinates can be seen in extended position of the leg. In the mechanism $\alpha_{i}, l_{i},(i=1,2,3)$ can be adjustable optionally according to the special geometry of knee joint. On the basis of published recommendations next data are proper approaching: $\alpha_{1}=\alpha_{2}=90^{\circ}$, $\alpha_{3}=0^{\circ}, l_{1}=l_{2}=l_{3}=0$.

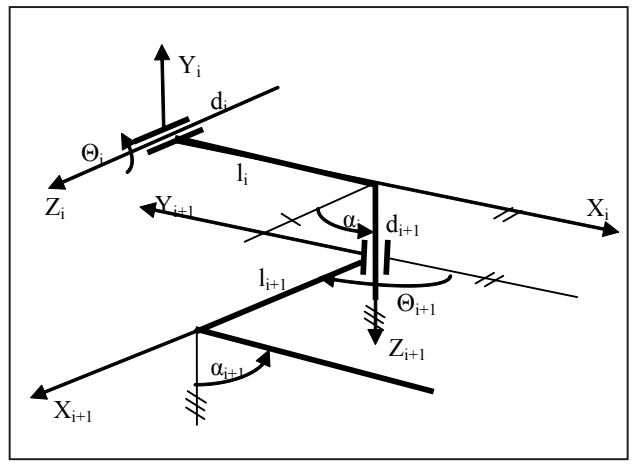

Figure 4. Connection of $i$ th and $i+1$ th bodies and joined coordinate-systems 
The application of the model enables the calculation of following quantities:

- $\Theta_{1}$ - flexion, in drawn position 0 degree,

$-\Theta_{2}-$ ab/adduction, in drawn position 90 degree,

$-\Theta_{3}$ - rotation of the tibia, in drawn position 0 degree,

$-d_{1}, d_{2}, d_{3}-$ moving on accordant axes.

On the basis of approaching $\alpha_{1}=\alpha_{2}=90^{\circ}$, $\alpha_{3}=0^{\circ}, l_{1}=l_{2}=l_{3}=0$ for the kinematical chain of Figure 3 the following matrix equation can be written down where $\mathbf{n}, \mathbf{o}, \mathbf{a}$ are unit vectors of coordinate-system $X_{3} Y_{3} Z_{3}$ in coordinatesystem $X_{0} Y_{0} Z_{0}$ and $P_{x^{\prime}} P_{y^{\prime}} P_{z}$ are coordinates of origin of coordinate-system $X_{3} Y_{3} Z_{3}$ in coordinate-system $X_{0} Y_{0} Z_{0}$.

$\left[\begin{array}{cccc}\cos \Theta_{1} & 0 & \sin \Theta_{1} & 0 \\ \sin \Theta_{1} & 0 & -\cos \Theta_{1} & 0 \\ 0 & 1 & 0 & d_{1} \\ 0 & 0 & 0 & 1\end{array}\right] *\left[\begin{array}{cccc}\cos \Theta_{2} & 0 & \sin \Theta_{2} & 0 \\ \sin \Theta_{2} & 0 & -\cos \Theta_{2} & 0 \\ 0 & 1 & 0 & d_{2} \\ 0 & 0 & 0 & 1\end{array}\right] *$

$*\left[\begin{array}{cccc}\cos \Theta_{3} & -\sin \Theta_{3} & 0 & 0 \\ \sin \Theta_{3} & \cos \Theta_{3} & 0 & 0 \\ 0 & 0 & 1 & d_{3} \\ 0 & 0 & 0 & 1\end{array}\right]=\left[\begin{array}{cccc}n_{x} & o_{x} & a_{x} & P_{x} \\ n_{y} & o & a_{y} & P_{y} \\ n_{z} & o & a_{z} & P_{z} \\ 0 & 0 & 0 & 1\end{array}\right]$

Roots of equation system: $\Theta_{1}, \Theta_{2}, \Theta_{3}, d_{1}, d_{2}$, $d_{3}$, which determine precisely the position of tibia compared to femur.

\section{Sensitivity investigation of results}

As results obtained kinematical parameters and diagrams depend partly on method of measurement and anatomical specialties cadaver however principally on determination of coordinate-systems joined to femur and tibia.

Considering the irregular shapes of femur and tibia the anatomical landmarks can be determined with more or less position mistake for this reason the anatomical coordinate-systems joined to anatomical landmarks have more or less position mistake as well ${ }^{15}$. The following anatomical landmarks must be measured:

- centre of the femoral head $(f h)$,

- medial and lateral epicondyles $\left(P_{m c l}, P_{l c l}\right)$,

- apex of the head of the fibula $(h f)$,

- prominence of the tibial tuberosity $(t t)$,

- distal apex of the lateral and medial malleolus $(k b, b b)$.

The optical positioning of above mentioned anatomical landmarks was made by a special appliance using pointer. The position mistakes of middle point of femur, apex of the head of the fibula and prominence of the tibial tuberosity cause small angular mistakes because these point are located relative far from origins of coordinate-systems.

The origins of coordinate-systems are determined between epicondyles and apices of the lateral and medial malleolus therefore the effect of position mistakes on position and orientation of coordinate-systems is quite significant. Considering the different shapes of investigated cadaver femur and tibia and position mistakes of measurements - depending on cadaver ones - more or less different kinematical functions can be obtained.

As first step of sensitivity investigation the positions of epicondyles were modified in coordinate-system $f s$ step by step $( \pm 2 \mathrm{~mm}$ ) approximately in the vertical plane (Figure 1). In second phase the position of apices of the lateral and medial malleolus were modified in coordinate-system $t s$ step by step $( \pm 2 \mathrm{~mm}$ ) approximately in the vertical plane as well (Figure 2). Difference from basic function (thickened curve) can be seen in Figure 5-6. (The basic function contains some mistakes because it was calculated from measured data.) 

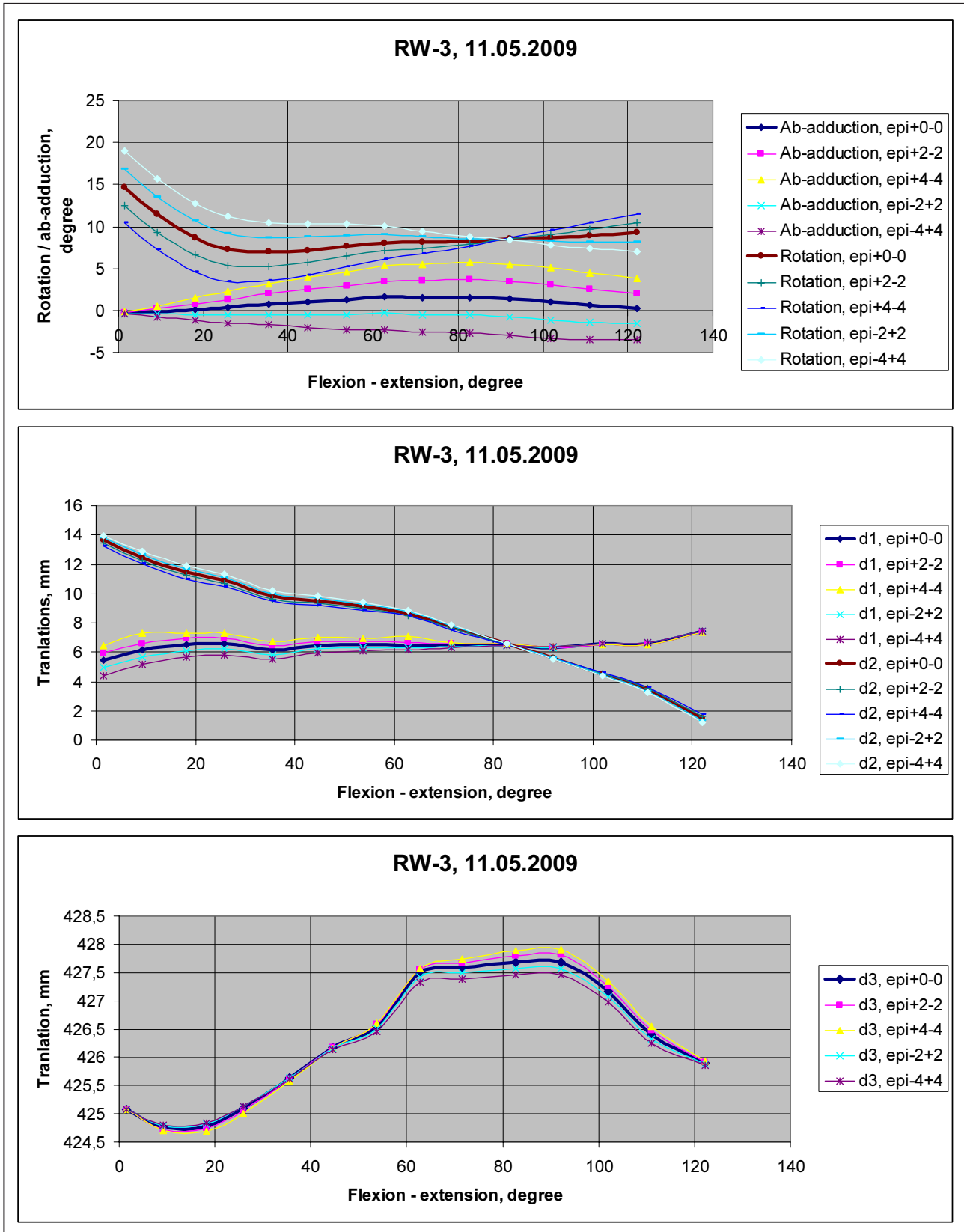

Figure 5. Effect of modification of position of epicondyles on kinematical functions 

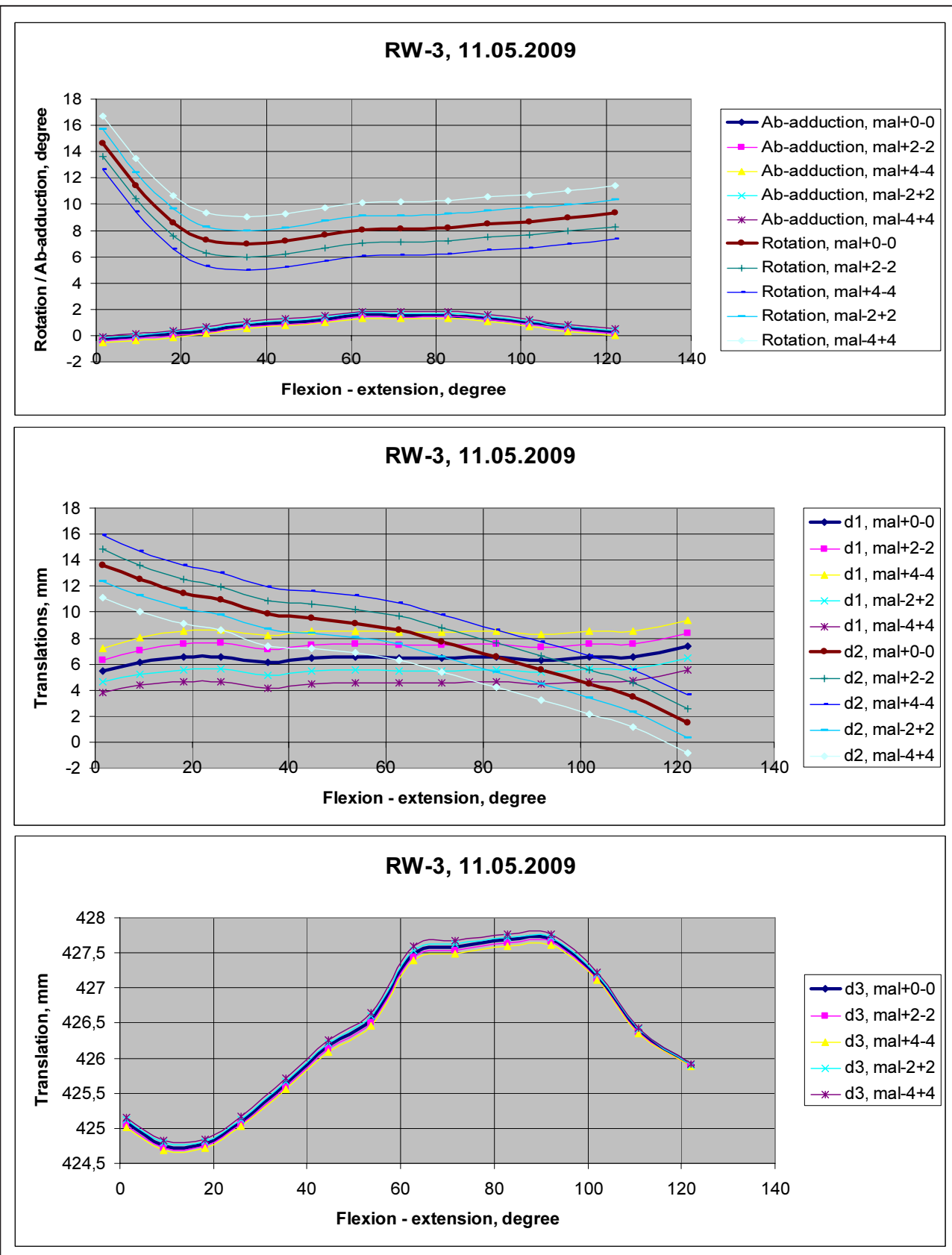

Figure 6. Effect of modification of position of apices of the lateral and medial malleolus on kinematical functions 


\section{Conclusion}

As result of sensitivity investigation the followings are established:

- The flexion-rotation and flexion-ab/adduction diagrams depend strongly on position mistakes of determined coordinate-systems, for this reason it is important to determine the anatomical landmarks.

- The cause of modification of diagrams is first of all the position mistake in direction of $Y$ axis of epicondyles.

- Its effect can be seen over 60 degree in flexed position. In this part the diagrams are approximately linear, their gradient vari- able depending on position mistakes of epicondyles.

- The shapes of ab/adduction diagrams are strongly deformed.

Summing up it can be established that the using of anatomical coordinate-systems enables the comparison and generalization of results of different motion investigation of human knee joints. On the basis of above mentioned it can be decided which parts of obtained results are acceptable certainly which parts and its conclusions need chary treatment. By more precise prescription of taking up of anatomical coordinate-system the comparison of results can be intensified.

\section{REFERENCES}

1. Grood ES, Suntay WJ. A joint coordinate system for the clinical description of three-dimensional motions: application to the knee. J. Biomech. Engng 1983;105: pp. 136-144.

2. Pennock GR, Clark KJ. An anatomybased coordinate system for the description of the kinematic displacements in the human knee. J. Biomechanics 1990; Vol. 23, No. 12: pp. 1209-1218.

3. Blankevoort L, Huiskes $R$, Lange A. Helical axes of passive knee joint motions. J. Biomechanics 1990; Vol. 23, No. 12, pp. 1219-1229.

4. Hollister AM, et al. The axes of rotation of the knee. Clinical Orthopaedics and Related Research 1993;(290): p. 259.

5. Fujie H, Livesay GA, Fujita M, Woo SLY. Forces and Moments in six-dof at the Human Knee Joint: Mathematical Description for Control. J. Biomechanics 1996; Vol. 29, No. 12:15771585.

6. Churchill DL, et al. The transepicondylar axis approximates the optimal flexion axis of the knee. Clinical Orthopaedics and Related Research, 1998;(356): p. 111-118.
7. Bull AMJ, Amis AA. Knee joint motion: description and measurement Proc Instn Mech Engrs 1998;Vol 212, Part H.

8. Iwaki H, Pinskerova V, Freeman MAR. Tibiofemoral movement 1 : the shapes and relative movements of the femur and tibia in the unloaded cadaver knee. The Journal of Bone and Joint Surgery [Br] 2000;82-B:1189-1195.

9. Wilson DR, Feikes JD, Zavatsky AB, O'Connor JJ. The components of passive knee movement are coupled to flexion angle. Journal of Biomechanics 2000;33:465-473.

10. Bíró I. Kinematical investigation of human knee joint. $21^{\text {th }}$ Working Meeting of the IFToMM Permanent Commission for Standardization of Terminology, Proceedings of the sientific seminar, June 27-July 2, 2005. Bardejov Spa, Slovakia, 91-96. p.

11. Szakál Z. Méróberendezés térdízület mozgásvizsgálatához. GÉP, 2006; LVII. évfolyam 1. szám: 37-40.

12. Katona G, M. Csizmadia B, Fekete G. Kísérleti vizsgálatok térd mechanikai modelljének meg- 
alkotásához. 2. Magyar Biomechanikai konferencia, Debrecen, 2006. június 30-július 1. p. $49-50$.

13. Bíró I, M. Csizmadia B, Katona G. New approximation of kinematical analysis of human knee joint. Bulletin of the Szent István University, ISSN 1586-4502 2008; p. 330-338

14. Bíró I. Az emberi térd három-hengeres kinematikai modellje. Elektronikus Múszaki Füze- tek VII. DAB Mûszaki Szakbizottság, ISBN 978-963-7064-22-7, Debrecen 2009; p. 107-112.

15. Katona G, M. Csizmadia B, Bíró I, Andrónyi K, Krakovits $G$. Cadaver térdek mozgásának értékelése anatómiai koordináta-rendszerek felhasználásával. Proceedings of $4^{\text {th }}$ Hungarian Conference on Biomechanics, Pécs, May 7-8, 2010.

16. http://www.ulb.ac.be/project/vakhum 